\title{
Derivation of the Equation Nernst-Aibassov in a Magnetic Field
}

\author{
Aibassov Yerkin Zhakenovich*, Yemelyanova Valentina, Tussupbayev Nessipbay, Shakieva Tatyana and \\ Yerzhanova Zhadyra \\ Research Institute of New Chemical Technologies and Materials, Kazakh National University Al-Farabi, Almaty 005012, Kazakhstan
}

Abstract: The influence of magnetic field on the redox potentials of the Nernst equation. The author offered the new formula Nernst equation in a magnetic field. Our proposed formula takes into account the influence of the magnetic field on the redox processes.

Key words: Nernst equation, magnetic field, redox processes.

\section{Introduction}

At present, the rapid development of science and technology and equipment allows us to look at the classical equations with new perspectives.

The actual problem is the question of how to lead the Nernst equation in the presence of a strong magnetic field. Addressing these issues is scientific and practical value.

The purpose of the work is to consider how to behave in the Nernst equation under the action of a magnetic field.

\section{Theory}

Nernst equation - an equation that relates the redox potential of the system with active substances included in the electrochemical equations, and standard electrode potentials of redox couples under the influence of a magnetic field [1-7].

\subsection{Conclusion Nernst Equation}

Nernst studied the behavior of electrolytes by passing an electric current and discovered the law. The law establishes the relationship between the electromotive force (potential difference) and ion

\footnotetext{
*Corresponding author: Aibassov Yerkin Zhakenovich, professor, research field: organic chemistry of $\mathrm{U}, \mathrm{Th}, \mathrm{As}, \mathrm{Sb}$, Bi. E-mail: erkin53@mail.ru.
}

concentration. As known, Nernst equation to predict the maximum operating potential, which may be prepared by the electrochemical interaction. Thus, this law relates to the thermodynamics of electrochemical theory in solving problems related to very dilute solutions.

$$
E=E^{0}+R T / n F \ln a_{O x} / a_{R e d}
$$

where, $E$-electrode potential; $E^{0}$ —standard electrode potential, V; $R$-universal gas constant equal, 8.31 $\mathrm{J} /(\mathrm{mol} \cdot \mathrm{K}) ; T$-absolute temperature, K; $F$-Faraday constant, $96,485.35 \mathrm{~mol}^{-1} ; n$ - the number of electrons involved in the process; $a_{O x}$ and $a_{R e}$ - activity respectively oxidized and reduced forms of the substances involved in the half reaction.

If the Nernst formula to substitute the numerical values of the constants $\mathrm{R}$ and $\mathrm{F}$, and go from natural logarithms to decimal, when $\mathrm{T}=298 \mathrm{~K}$ we obtain:

$$
E=E^{0}+0.0592 / n \lg a_{O x} / a_{R e d}
$$

\section{Results and Discussion}

To display the Nernst equation in a magnetic field is necessary to consider the Lorentz force.

Lorentz force-the force with which the electromagnetic field according to the classical (non-quantum) electrodynamics operates on a point charge. Lorentz force called the force acting on moving with velocity $v$ charge $q$ only by the electromagnetic 
field of the electric $E$ and magnetic $B$ fields.

$$
F=q(E+[v \times B])
$$

The direction of the Lorentz force and the direction of its deviation caused by a charged particle in a magnetic field depend on the sign of the charge $Q$ of the particle. The authors assume that the magnetic field is uniform and the particle electric fields do not work. If a charged particle moves in a magnetic field with velocity $\mathrm{v}$ along the lines of magnetic induction, the angle $a$ between the vectors $v$ and $B$ is 0 or p. Then the formula (3) the Lorentz force is equal to zero, i.e. The magnetic field on the particle does not work and it is moving uniformly and rectilinearly.

If a charged particle moves in a magnetic field with the velocity $v$, perpendicular to the vector $B$, the Lorentz force $F=Q[v B]$ is constant in magnitude and normal to the trajectory of the particle. According to second Newton's law, this force creates a centripetal acceleration. Hence it follows that the particle will move in a circle, the radius $\mathrm{r}$ is determined from the condition $Q v B=2 m v / r$.

where, the radius $r$,

$$
r=m / Q v / B
$$

The period of rotation of the particle, i.e. The time $T$ for which it makes one complete revolution,

$$
T=2 \pi r / v
$$

Substituting the expression (3), the authors obtain

$$
T=2 \pi / B m / Q
$$

the period of rotation of a particle in a uniform magnetic field is determined only by the reciprocal of the specific charge $(Q / m)$ particles and the magnetic induction field, but does not depend on its velocity (when $\mathrm{v}<<\mathrm{c}$ ). This is the basis of cyclic action of charged particle accelerators [8-11].

Thus, the Nernst equation in a magnetic field will be expressed by the Eqs. (6) or (7):

$$
E=E^{0}+R T / n F \ln a_{O x} / a_{R e d}+1 / 4 \pi H d B
$$

or

$$
E=E^{0}+0.0592 \ln \lg a_{O x} / a_{R e d}+1 / 4 \pi H d B
$$

\section{Conclusions}

The authors offered the new formula Nernst equation in a magnetic field. The proposed formula takes into account the influence of the magnetic field on the redox processes. The authors first discovered that the Nernst Equation in a magnetic field will be of the form:

$$
E=E^{0}+0.0592 / n \lg a_{O x} / a_{R e d}+1 / 4 \pi H d B
$$

\section{Acknowledgments}

The authors would like to thank Lynn C. Francesconi (Hunter College CUNY), Ruben M. Savizky (Columbia University, New York), Peter C. Burns (Notre Dame University, Indiana) and Chistopher L. Cahill (George Washington University) for discussion of the results.

\section{References}

[1] Orna, M. V., and Stock, J. 1989. Electrochemistry, past and present. Columbus, Ohio: American Chemical Society. ISBN 0-8412-1572-3.

[2] Wahl. 2005. "A Short History of Electrochemistry." Galvanotechtnik 96 (8): 1820-8.

[3] Zutshi, K. 2008. "Introduction to Polarography and Allied Techniques." The Journal of Physical Chemistry 112: $127-8$.

[4] Hutchings, G. J., Heneghan, C. S., Hudson, I. D., and Taylor, S. H. 1996. "Uranium-Oxide-Based Catalysts for the Destruction of Volatile Chloro-Organic Compounds." Nature 384: 341-43.

[5] Grasselli, R. K., and Callahan, J. L. 1969. "Structure-Catalytic Efficiency Relationships in U-Sb Oxide Acrylonitrile Synthesis Catalysts." J. Catal. 14: 93-103.

[6] Corberan, V. C., Kremenic, G., and Tejuca, G. 1988. "Acidity and Oxidation Activity of $\mathrm{MoO}_{3}-\mathrm{UO}_{3} / \mathrm{SiO}_{2}$ Catalysts." React. Kinet. Catal. Lett. 36: 235-40.

[7] Gordeeva, L. G., Aristov, Y. I., Moroz, E. M., Rudina, N. A., Zaikovskii, V. I., Tanashev, Y. Y., et al. 1995. "Preparation and Study of Porous Uranium Oxides as Supports for New Catalysts of Steam Reforming of Methane." J. Nucl. Mater. 218: 202-9.

[8] Aibassov, E. Z., and Umirkulova, Z. S. 2012. "In Getting the Uranium Catalyst based on Natural Zeolite Deposits Chankanayskogo." Proceedings of Symposium "Fronties 
of Organometallic Chemistry", Saint-Petersburg, Russia.

[9] Aibassov, E. Z., and Aibassova, S. M. 2011. "The New Reactions of Arsine." In Introduction to Organic Chemistry of Arsenic, Actinides, Lanthanides, $\mathrm{Os}^{187}$ and Re., p 100.

[10] Aibassov, Y. Z., and Yemelyanova, V. S. 2014. "Effect of Magnetic Field on the Catalysis of Ligands in Non-aqueous Solutions." Journal of Chemical Engineering and Chemistry Research 1 (2): 132-7.

[11] Aibassov, Y. Z., and Yemelyanova, V. S. 2014. Magnetic and Relativistic Effects in Catalysis and Uranium Catalysts, Almaty: Infiniline Publishing House, Inc., p 184. 